\title{
A deeper view of the CoRoT-9 planetary system
}

\section{A small non-zero eccentricity for CoRoT-9b likely generated by planet-planet scattering}

\author{
A. S. Bonomo ${ }^{1}$, G. Hébrard ${ }^{2,3}$, S. N. Raymond ${ }^{4}$, F. Bouchy ${ }^{5}$, A. Lecavelier des Etangs ${ }^{2}$, P. Bordé ${ }^{4}$, S. Aigrain ${ }^{6}$, \\ J.-M. Almenara ${ }^{5}$, R. Alonso ${ }^{7,8}$, J. Cabrera ${ }^{9}$, Sz. Csizmadia ${ }^{9}$, C. Damiani ${ }^{10}$, H. J. Deeg ${ }^{7,8}$, M. Deleuil ${ }^{11}$, R. F. Díaz ${ }^{12,13}$, \\ A. Erikson ${ }^{9}$, M. Fridlund ${ }^{14,15}$, D. Gandolfi ${ }^{16}$, E. Guenther ${ }^{17}$, T. Guillot ${ }^{18}$, A. Hatzes ${ }^{17}$, A. Izidoro ${ }^{19,4}$, C. Lovis $^{5}$, \\ C. Moutou ${ }^{20,11}$, M. Ollivier ${ }^{10,21}$, M. Pätzold ${ }^{22}$, H. Rauer ${ }^{9}, 23$, D. Rouan ${ }^{21}$, A. Santerne ${ }^{11}$, and J. Schneider ${ }^{24}$ \\ (Affiliations can be found after the references)
}

Received 15 February 2017 / Accepted 17 March 2017

\begin{abstract}
CoRoT-9b is one of the rare long-period ( $P=95.3$ days) transiting giant planets with a measured mass known to date. We present a new analysis of the CoRoT-9 system based on five years of radial-velocity (RV) monitoring with HARPS and three new space-based transits observed with CoRoT and Spitzer. Combining our new data with already published measurements we redetermine the CoRoT-9 system parameters and find good agreement with the published values. We uncover a higher significance for the small but non-zero eccentricity of CoRoT-9b $\left(e=0.133_{-0.037}^{+0.042}\right)$ and find no evidence for additional planets in the system. We use simulations of planet-planet scattering to show that the eccentricity of CoRoT- $9 \mathrm{~b}$ may have been generated by an instability in which a $\sim 50 M_{\oplus}$ planet was ejected from the system. This scattering would not have produced a spin-orbit misalignment, so we predict that the CoRoT-9b orbit should lie within a few degrees of the initial plane of the protoplanetary disk. As a consequence, any significant stellar obliquity would indicate that the disk was primordially tilted.
\end{abstract}

Key words. planetary systems - techniques: radial velocities - techniques: photometric - stars: individual: CoRoT-9

\section{Introduction}

As of February 2017, only 27 warm Jupiters (WJs), defined as giant planets $\left(M_{\mathrm{p}}>0.1 M_{\mathrm{Jup}}\right)$ with orbital distance $0.1<a<1 \mathrm{AU}$ (e.g. Dawson \& Chiang 2014), are known to transit in front of their host stars and to have a measured mass better than $3 \sigma^{1}$. All except four of these WJs were discovered by space-based missions as, in general, only these surveys provide photometric time series of sufficient precision, length, and sampling consistency to detect them. Indeed, the first WJ detected via the transit method was discovered by the CoRoT space telescope; this object, called CoRoT-9b, orbits a non-active G3V star with orbital period $P=95.3 \mathrm{~d}$ and semimajor axis $a=0.41 \mathrm{AU}$, and has a mass of $0.84 \pm 0.07 M_{\text {Jup }}$ and a radius of $1.05 \pm 0.04 R_{\text {Jup }}$ (Deeg et al. 2010, hereafter D10). Thanks to the Kepler space mission, many more WJ candidates could be found and, for some of them, radial-velocity (RV) follow up (e.g. Santerne et al. 2012, 2016, and references therein) and/or analysis of transit time variations (e.g. Dawson et al. 2012; Borsato et al. 2014; Bruno et al. 2015) have allowed both to unveil their planetary nature and determine their mass and hence their densities. Specifically, 18 of the aforementioned 27 transiting WJs were discovered by Kepler.

The discovery and characterisation of transiting WJs are of great importance to better understand the internal structure, formation, and evolution of giant planets. For instance, the mass-radius relation of giant planets at orbital distances $a>$ $0.1 \mathrm{AU}$ should not be affected by stellar heating, which is likely related to the inflation mechanism responsible for the large radii

\footnotetext{
1 Data from http://exoplanetarchive.ipac.caltech.edu and
} http://exoplanet.eu of several hot Jupiters (Schneider et al. 2011; Demory \& Seager 2011; Sozzetti et al. 2015). Therefore, WJs are not expected to be inflated unless other processes are at work.

The formation and orbital evolution of WJs are currently a very interesting issue of debate. None of the processes that have been invoked to explain the population of close-in giant exoplanets obviously applies to WJs. Inward type II migration (e.g. Lin et al. 1996) halted by disk photoevaporation may produce WJs (e.g. Alexander \& Pascucci 2012; Mordasini et al. 2012), but this migration cannot explain the high eccentricities of many of them (Bonomo et al. 2017) given that migration in the disk only tends to damp non-zero eccentricities (e.g. Kley \& Nelson 2012). In addition, planet-planet scattering becomes less effective closer to the star (Petrovich et al. 2014). Based on a population-synthesis study, Petrovich \& Tremaine (2016) found that $\sim 20 \%$ of WJs may have migrated through high-eccentricity migration (e.g. Rasio \& Ford 1996), in particular through the high-eccentricity phase of secular oscillations excited by an outer companion in an eccentric and/or mutually inclined orbit (see also Wu \& Lithwick 2011). On the contrary, Hamers et al. (2017) were not able to produce any WJ from secular evolution in multi-planet systems with three to five giant planets and suggested that WJs either underwent disk migration or formed in situ. In situ formation is proposed by Huang et al. (2016) as the most likely mechanism to form multi-planet systems with WJs flanked by close and smaller companions, such as those observed by Kepler. The rate of occurrence of WJs in such systems may be relatively high, up to $\sim 50 \%$, although it is highly uncertain at the moment (Huang et al. 2016). The same authors argued that the WJs without known close companions might represent a distinct population that formed and migrated differently 
from the former population (e.g. WJs in compact multi-planet systems).

Yet, it is also possible that WJs with no close-in small companions are simply the innermost planetary cores of the system that grew into gas giants and migrated inward. The birth and migration of a gas giant play a crucial role in the evolution and dynamics of a young planetary system. In this context, two aspects can help to explain the lack of (detected) inner companions in these specific systems. The first aspect is that a (forming) gas giant stops the radial flux of small planetesimal and pebbles drifting inward due to gas drag (Lambrechts et al. 2014). This could cause the region inside the orbit of the putative growing and migrating WJ to be too low mass to support, for instance, the formation of any planet larger than the Earth. Secondly, a gas giant formed from the innermost planetary core in the system also acts as an efficient dynamical barrier to additional inward-migrating planetary cores (or planets) formed on outer orbits (Izidoro et al. 2015). Thus, inward-migrating planets from external parts of the disk typically cannot make their full way inward to the innermost regions. They tend to be captured in external mean motion resonances with the gas giant. This could also help to explain the lack of inner companions in these systems. On the other hand, one should naturally expect that outer companions to WJs should be common in this context. However, the subsequent dynamical evolution of these planetary systems post-gas dispersal (e.g. occurrence of dynamical instabilities or not) is determinant in setting the real destiny of these planets.

Monitoring of planetary systems containing WJs that are not flanked by close and small companions, with RV and/or (Gaia) astrometric measurements as well as observations of adaptive optics imaging are crucial to i) search for outer (planetary or stellar) companions; ii) provide information on whether these exterior companions may have triggered high-eccentricity migration (e.g. Bryan et al. 2016); and iii) detect significant orbital eccentricities that could be an imprint of secular chaos or planetplanet scattering possibly occurring after early disk migration (e.g. Marzari et al. 2010).

CoRoT-9b belongs to the class of WJs with no detected close transiting companions. In this work we present photometric follow-up with CoRoT and Spitzer (Sect. 2.1) and spectroscopic monitoring with HARPS for a total time span of almost five years (Sect. 2.2). With a combined Bayesian analysis of photometric and RV data (Sect. 3), we redetermine the system parameters and uncover a higher significance for the small eccentricity of CoRoT-9b (Sect. 4.1). We find no evidence for additional planetary companions in the system with the gathered data (Sect. 4.2). Finally, we investigate several scenarios for the possible formation and migration of CoRoT-9b (Sect. 5.1) and carry out planet-planet scattering simulations to reconstruct the dynamical history of the CoRoT-9 planetary system that best matches the observational constraints (Sect. 5.2).

\section{Data}

\subsection{Photometric data}

In addition to one full and one partial transit of CoRoT-9b observed by the CoRoT satellite in 2008 (D10), here we make use of two more transits: the first was observed by Spitzer on 18 June 2010 and the second on 4 July 2011 simultaneously by Spitzer and CoRoT (see Fig. 1). The temporal sampling of CoRoT and Spitzer data is 32 and 31 s, respectively.

CoRoT photometry with the imagette pipeline (e.g. Barros et al. 2014) was obtained during the CoRoT SRc03
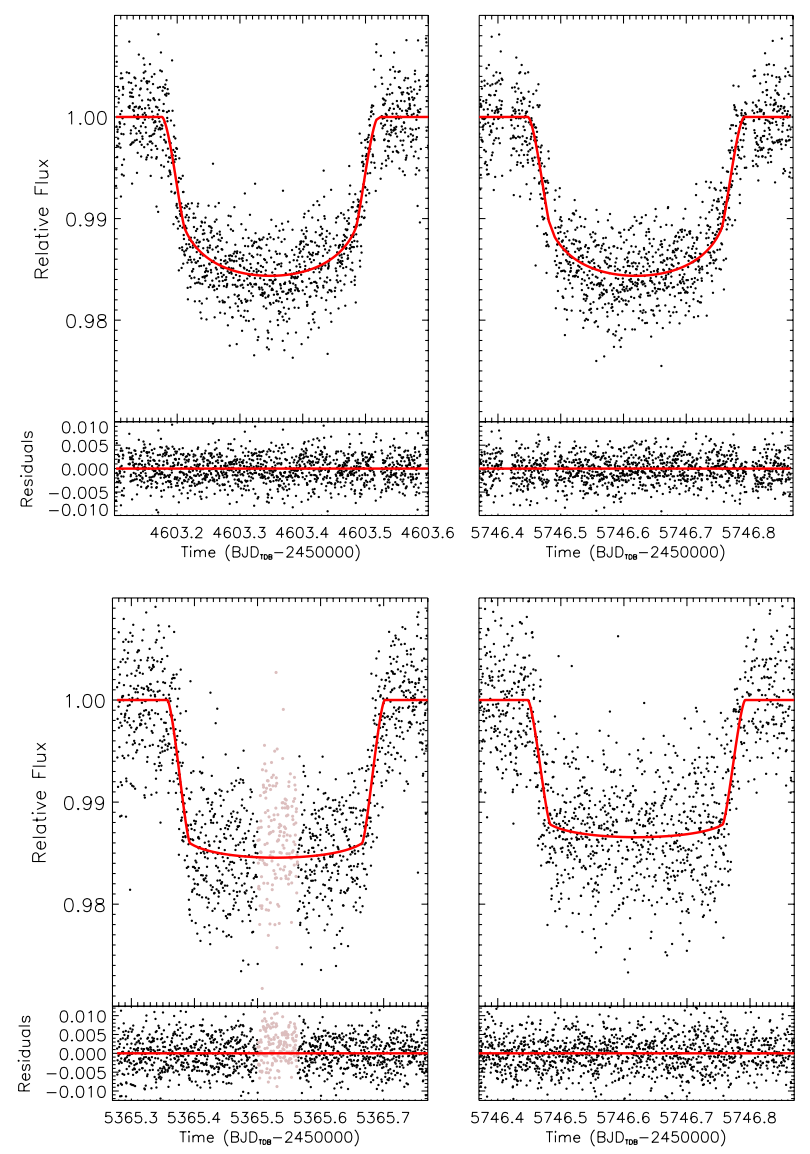

Fig. 1. Top panel: CoRoT-9b full transits as observed by the CoRoT satellite with a $32 \mathrm{~s}$ cadence (optical band) and the best-fit model (red solid line). Bottom panel: the other two transits observed with Spitzer at $4.5 \mu \mathrm{m}$ with a $31 \mathrm{~s}$ cadence along with the transit model (red solid line). We note the different transit depths and the "bump" at the middle of the first transit (grey points), which we attribute to uncorrected systematics affecting the 2010 Spitzer transit (see text).

pointing ${ }^{2}$, which lasted only five days and was dedicated to the observation of the CoRoT-9b transit. Flux contamination by background stars in the CoRoT mask (Llebaria \& Guterman 2006; Deeg et al. 2009) was estimated to be very low, that is $2.5 \%$, by D10 for the data acquired in 2008; we adopted the same value. However, the imagette pipeline does not permit us to estimate such a contamination for the transit observations in 2011 as well; hence we included a dilution factor as an additional free parameter in the transit fitting (Sect. 3).

Both Spitzer observations were secured with the Channel 2 at $4.5 \mu \mathrm{m}$ of the IRAC camera (Fazio et al. 2004). These infrared observations and their reduction are described in Lecavelier des Etangs et al. (2017) who present a search for signatures of rings and satellites around CoRoT-9b. Here we use those Spitzer light curves to refine the parameters of CoRoT$9 \mathrm{~b}$ and its host star. Briefly, we used the basic calibrated data files of each image as produced by the IRAC pipeline, then corrected them for the so-called pixel-phase effect, which is the oscillation of the measured flux due to the Spitzer jitter and the detector intra-pixel sensitivity variations. Despite this correction, the 2010 Spitzer transit light curve shows two residual systematic effects (Lecavelier des Etangs et al. 2017): first, it presents a "bump" near the middle of the transit, which is unlikely to be

2 Data available at the CoRoT archive: http://idoc-corot.ias. $\mathrm{u}$-psud. fr 
due to the crossing of a large starspot by the planet given that the host star is very quiet (no activity features are seen in the CoRoT light curve); secondly, the transit is significantly deeper than that observed in 2011 by $\sim 13 \%$ (see Fig. 1 and Sect. 4.1). This larger depth cannot be attributed to unocculted starspots because it would imply a spot filling factor of $\sim 40 \%{ }^{3}$ that is again unrealistic for the CoRoT-9 low activity level. To overcome these effects, we excluded from our analysis the data points in the bump and, similarly to the 2011 CoRoT transit, we considered a dilution factor for the 2010 Spitzer data to account for its larger depth (see Sect. 3). By doing so, we substantially rely on the 2011 Spitzer transit for the determination of the planetary radius at $4.5 \mu \mathrm{m}$ because this transit does not show any feature that might be related to residual systematic effects.

CoRoT and Spitzer transit light curves were normalised following Bonomo et al. (2015); we excluded the partial CoRoT transit for the determination of system parameters (Sect. 3) because of a possible non-optimal normalisation due to the lack of egress data, but we used it for the computation of transit timing variations (TTVs) (Sects. 3 and 4.2). Correlated noise on an hourly timescale in each light curve was estimated as in Aigrain et al. (2009) and Bonomo et al. (2012) but was found to be practically negligible for all the transits. After subtracting the transit model (Sect. 4.1), the CoRoT data have an rms of $\sim 2.8 \times 10^{-3}$ in units of relative flux while the Spitzer measurements show a higher rms of $\sim 4.5 \times 10^{-3}$; there is no significant difference in the rms among the CoRoT transits or between the two Spitzer transits.

We also searched for additional transit signals in the CoRoT data with the LAM pipeline (Bonomo et al. 2012; see also Bonomo et al. 2009) after removing the CoRoT-9b transits, but found none (see Sect. 4.2).

\subsection{Radial-velocity data}

We obtained 28 radial-velocity observations of CoRoT-9 between September 2008 and August 2013 with the HARPS fibre-fed spectrograph (Mayor et al. 2003) at the $3.6 \mathrm{~m}$ ESO telescope in La Silla, Chile (programme 184.C-0639). The resolution power is 115000 . Depending on the observations, exposure times range between 40 and 60 min and provide signal-to-noise ratios between 11 and 24 per pixel at $550 \mathrm{~nm}$. All the observations were gathered with the high-accuracy mode (HAM) of HARPS except that at $\mathrm{BJD}_{\mathrm{UTC}}=2454766.51$, which was secured in high-efficiency (EGGS) mode; we decided to keep this observation in our analysis as it shows no significant drift in radial velocity.

The spectra were extracted using the HARPS pipeline and the radial velocities were measured from the weighted cross-correlation with a numerical mask (Baranne et al. 1996; Pepe et al. 2002). We tested masks representative of F0, G2, and K5 stars. The bluest of the 68 HARPS spectral orders are noisy for that relatively faint $(V=13.7)$ star so we adjusted the number of orders used in the cross-correlation. The solution we adopt is the cross-correlation performed with the K5-type mask with the exclusion of the 10 first blue orders. We chose that configuration because it minimises the dispersion of the RV residuals after the Keplerian fit. Other configurations do not provide significantly different system parameters, but their residual dispersions are larger. Following the method presented in Estimated from Eq. (7) in Ballerini et al. (2012) by considering an
early G-type star and the $4.5 \mu \mathrm{m}$ Spitzer band (see their Tables 1 and 2).
Table 1. HARPS radial-velocity measurements of CoRoT-9.

\begin{tabular}{|c|c|c|c|c|c|}
\hline $\begin{array}{l}\text { BJD }_{\text {UTC }} \\
-2450000\end{array}$ & $\begin{array}{c}\mathrm{RV} \\
{\left[\mathrm{km} \mathrm{s}^{-1}\right]}\end{array}$ & $\begin{array}{c} \pm 1 \sigma \\
{\left[\mathrm{km} \mathrm{s}^{-1}\right]}\end{array}$ & $\begin{array}{l}\text { Bisect. }^{\dagger} \\
{\left[\mathrm{km} \mathrm{s}^{-1}\right]}\end{array}$ & $\begin{array}{c}T_{\exp }{ }^{\star} \\
\text { [s] }\end{array}$ & $S / N^{\star \star}$ \\
\hline 4730.5481 & 19.785 & 0.006 & -0.003 & 2700 & 18.3 \\
\hline 4734.5653 & 19.797 & 0.008 & -0.008 & 2700 & 15.7 \\
\hline 4739.5092 & 19.790 & 0.008 & 0.022 & 2700 & 16.1 \\
\hline $4754.5002^{\dagger \dagger}$ & 19.811 & 0.011 & 0.021 & 3600 & 19.1 \\
\hline 4766.5131 & 19.838 & 0.008 & -0.001 & 3600 & 17.3 \\
\hline 4771.5094 & 19.865 & 0.014 & 0.036 & 3100 & 10.6 \\
\hline $4987.7410^{\dagger \dagger}$ & 19.802 & 0.012 & 0.021 & 3600 & 16.0 \\
\hline $4993.8179^{\dagger \dagger}$ & 19.787 & 0.007 & 0.000 & 3600 & 22.8 \\
\hline $5021.6567^{\dagger \dagger}$ & 19.798 & 0.009 & 0.028 & 3600 & 19.7 \\
\hline $5048.6495^{\dagger \dagger}$ & 19.850 & 0.007 & 0.030 & 3600 & 21.8 \\
\hline 5063.5679 & 19.856 & 0.011 & -0.006 & 3600 & 16.5 \\
\hline 5069.5573 & 19.849 & 0.006 & 0.008 & 3300 & 18.7 \\
\hline $5077.5704^{\dagger \dagger}$ & 19.810 & 0.012 & -0.002 & 3000 & 16.0 \\
\hline 5095.5408 & 19.789 & 0.006 & 0.011 & 3600 & 19.7 \\
\hline 5341.9076 & 19.856 & 0.005 & 0.002 & 3600 & 23.3 \\
\hline $5376.7009^{\dagger \dagger}$ & 19.787 & 0.014 & -0.016 & 3600 & 16.5 \\
\hline $5400.6811^{\dagger \dagger}$ & 19.787 & 0.009 & 0.030 & 3600 & 20.3 \\
\hline 5413.6660 & 19.800 & 0.005 & 0.006 & 3600 & 24.0 \\
\hline 5423.6248 & 19.833 & 0.009 & -0.026 & 3600 & 15.9 \\
\hline $5428.5913^{\dagger \dagger}$ & 19.839 & 0.015 & -0.048 & 3600 & 13.7 \\
\hline 5658.8885 & 19.783 & 0.011 & -0.017 & 3600 & 13.5 \\
\hline 5682.9153 & 19.779 & 0.006 & 0.022 & 2400 & 20.9 \\
\hline 5713.8266 & 19.855 & 0.009 & 0.026 & 3600 & 15.7 \\
\hline $5769.5810^{\dagger \dagger}$ & 19.794 & 0.009 & -0.005 & 3600 & 14.9 \\
\hline 5824.5177 & 19.862 & 0.007 & 0.004 & 3600 & 19.4 \\
\hline 6120.6337 & 19.838 & 0.011 & 0.003 & 3600 & 12.8 \\
\hline 6469.6810 & 19.807 & 0.016 & 0.009 & 2700 & 13.9 \\
\hline 6515.6036 & 19.802 & 0.008 & 0.006 & 3600 & 17.2 \\
\hline
\end{tabular}

Notes. ${ }^{(\dagger)}$ Bisector spans; error bars are twice those of the RVs. ${ }^{(\star)}$ Duration of each individual exposure. ${ }^{(\star \star)}$ Signal-to-noise ratio per pixel at $550 \mathrm{~nm} .{ }^{(\dagger \dagger)}$ Measurements corrected for moonlight pollution.

Bonomo et al. (2010), moonlight contamination was corrected for 10 observations using the second optical-fibre aperture targeted on the sky. The corrections are of the order of $10 \mathrm{~m} \mathrm{~s}^{-1}$ or less, except for the two most polluted exposures where they are between 25 and $50 \mathrm{~m} \mathrm{~s}^{-1}$.

The RV measurements are reported in Table 1 and shown in Fig. 2. These measurements show variations in phase with the transit ephemeris derived from CoRoT and Spitzer photometry. The bisector spans of the cross-correlation function show neither variations nor trends as a function of radial velocity, confirming that CoRoT-9b is a well-secured planet.

The 28 HARPS measurements we present here have an average precision of $9 \mathrm{~m} \mathrm{~s}^{-1}$ and cover a time span of almost five years. This is a significant improvement compared with the 14 measurements gathered by D10 in a one-year time span. The 28 observations presented in Table 1 include those of D10, but with slightly different numerical values as our data reduction is not exactly identical. 

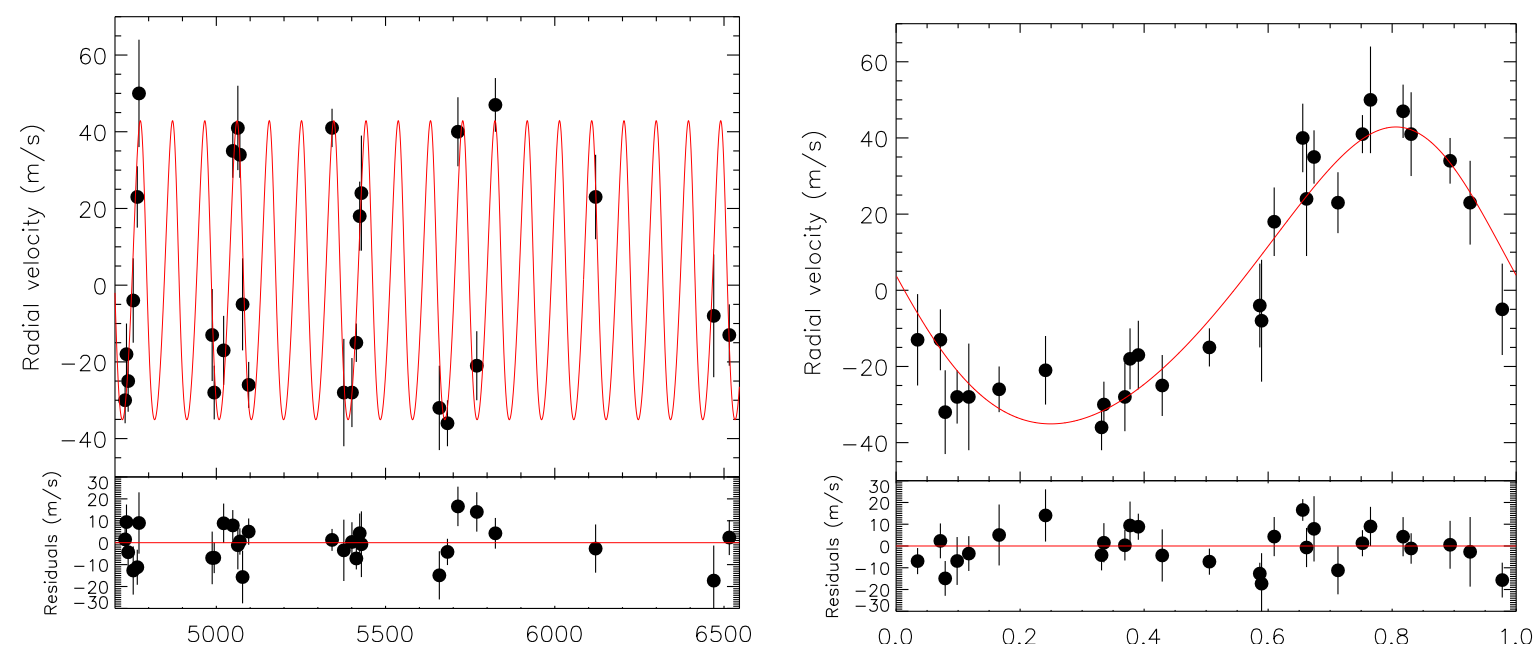

Fig. 2. Left panel: HARPS RVs of CoRoT-9 as a function of time and the best Keplerian model (red solid line). Right panel: the same as the left panel but as a function of the orbital phase (transits occur at phase equal to zero/one).

\section{Bayesian data analysis}

To derive the system parameters, we carried out a Bayesian combined analysis of the space-based photometric data and groundbased HARPS RVs, using a differential evolution Markov chain Monte Carlo (DE-MCMC) technique (ter Braak 2006; Eastman et al. 2013). For this purpose, the epochs of the photometric and spectroscopic data were converted into the same unit $\mathrm{BJD}_{\mathrm{TDB}}$ (Eastman et al. 2010); we used Eq. (4) in Shporer et al. (2014) to perform this correction for the Spitzer data. Given the relatively large semimajor axis of CoRoT-9b, light travel time between the transit measurements and the stellar-centric frame (to which RV epochs are referred) amounts to $\sim 3$ min and was taken into account to have all the data in the same reference frame (the system barycentre).

Our model consists of i) the Keplerian orbit to fit the RVs of the host star and ii) the CoRoT-9b transit model, for which we used the formalism of Mandel \& Agol (2002). The free parameters are as follows: the transit epoch $T_{0}$; the orbital period $P$; the systemic radial velocity $V_{\mathrm{r}}$; the radial-velocity semi-amplitude $K ; \sqrt{e} \cos \omega$ and $\sqrt{e} \sin \omega$ (e.g. Anderson et al. 2011); a RV jitter term $s_{\mathrm{j}}$ added in quadrature to the formal error bars to account for possible extra noise in the RV measurements; the transit duration from first to fourth contact $T_{14}$; the ratios of the planetary-tostellar radii $R_{\mathrm{p}} / R_{*}$ for both the CoRoT and Spitzer bandpasses; the inclination $i$ between the orbital plane and the plane of the sky; the two limb-darkening coefficients (LDC) $q_{1}=\left(u_{\mathrm{a}}+u_{\mathrm{b}}\right)^{2}$ and $q_{2}=0.5 u_{\mathrm{a}} /\left(u_{\mathrm{a}}+u_{\mathrm{b}}\right)$ (Kipping 2013), where $u_{\mathrm{a}}$ and $u_{\mathrm{b}}$ are the coefficients of the limb-darkening quadratic law ${ }^{4}$, for both the CoRoT and Spitzer bandpasses; and two contamination factors, one for the CoRoT transit observed in 2011 in imagette mode (see Sect. 2.1) and the other for the Spitzer transit observed in 2010 to account for the significant difference in depth with respect to the 2011 Spitzer transit (Sect. 2.1). Uniform priors were set on all parameters, in particular with bounds of $[0,1]$ for $q_{1}$ and $q_{2}$ (Kipping 2013), with a lower limit of zero for $K$ and $s_{\mathrm{j}}$, and an upper bound of 1 for the eccentricity; the lower limit of zero simply comes from the choice of fitting $\sqrt{e} \cos \omega$ and $\sqrt{e} \sin \omega$. Transit fitting was also performed for each individual

\footnotetext{
${ }^{4} I(\mu) / I(1)=1-u_{\mathrm{a}}(1-\mu)-u_{\mathrm{b}}(1-\mu)^{2}$, where $I(1)$ is the specific intensity at the centre of the disk and $\mu=\cos \gamma, \gamma$ being the angle between the surface normal and the line of sight.
}

transit to compute transit timing variations (Sect. 4.2) by fixing $e$ and $\omega$ to the values found with the combined analysis and by imposing a Gaussian prior on transit duration for the partial CoRoT transit.

The DE-MCMC posterior distributions of the model parameters were determined by i) maximising a Gaussian likelihood function (see e.g. Eqs. (9) and (10) in Gregory 2005); ii) adopting the Metropolis-Hastings algorithm to accept or reject the proposed steps; and iii) following the prescriptions given by Eastman et al. (2013) for the employed number of chains (twice the number of free parameters), the removal of burn-in steps, and the criteria for convergence and proper mixing of the chains. As usual, the medians and the $15.86 \%$ and $84.14 \%$ quantiles of the posterior distributions are taken as the best values and $1 \sigma$ uncertainties of the fitted and derived parameters. For parameters consistent with zero, we provide the $1 \sigma$ upper limits computed as the $68.27 \%$ confidence intervals starting from zero.

The stellar density as derived from the transit fitting, along with the effective temperature and metallicity of CoRoT-9 that are reported in D10, were interpolated to the Yonsei-Yale evolutionary tracks (Demarque et al. 2004) to find the most likely stellar, hence planetary, parameters and their associated uncertainties (e.g. Sozzetti et al. 2007; Bonomo et al. 2014).

\section{Results}

\subsection{System parameters}

Fitted and derived system parameters and their $1 \sigma$ error bars are given in Table 2. They are fully consistent, that is within $1 \sigma$, with those reported by D10. Figures 1 and 2 show the best-fit models to the full CoRoT and Spitzer transits and the RV data, respectively.

The values of $R_{\mathrm{p}} / R_{*}$ in the CoRoT and Spitzer bandpasses agree within $1 \sigma$, the former being slightly higher. Flux contamination by background stars in the CoRoT imagette mode was found to be negligible, that is $<0.4 \%$; the dilution factor for the first Spitzer transit to account for its larger depth (Sect. 2.1) is $13.2 \pm 1.5 \%$. The fitted limb-darkening coefficients agree well with the theoretical values computed by Claret \& Bloemen (2011) both for the CoRoT and Spitzer bandpasses.

One of the most remarkable results of our analysis is that, by doubling the number of collected HARPS RVs, the significance 
A. S. Bonomo et al.: A deeper view of the CoRoT-9 planetary system

Table 2. CoRoT-9 system parameters.

\begin{tabular}{|c|c|}
\hline \multicolumn{2}{|l|}{ Stellar parameters } \\
\hline Stellar mass $\left[M_{\odot}\right]$ & $0.96 \pm 0.04$ \\
\hline Stellar radius $\left[R_{\odot}\right]$ & $0.96 \pm 0.06$ \\
\hline Stellar density $\rho_{*}\left[\mathrm{~g} \mathrm{~cm}^{-3}\right]$ & $1.51 \pm 0.30$ \\
\hline Age $t[\mathrm{Gyr}]$ & $6 \pm 3$ \\
\hline Effective temperature $T_{\text {eff }}[\mathrm{K}]^{a}$ & $5625 \pm 80$ \\
\hline Stellar surface gravity $\log g[\mathrm{cgs}]^{a}$ & $4.54 \pm 0.09$ \\
\hline Stellar metallicity $[\mathrm{Fe} / \mathrm{H}][\mathrm{dex}]^{a}$ & $-0.01 \pm 0.06$ \\
\hline Stellar rotational velocity $V \sin i_{*}\left[\mathrm{~km} \mathrm{~s}^{-1}\right]^{a}$ & $<3.5$ \\
\hline CoRoT limb-darkening coefficient $q_{1}$ & $0.40_{-0.10}^{+0.13}$ \\
\hline CoRoT limb-darkening coefficient $q_{2}$ & $0.33_{-0.10}^{+0.13}$ \\
\hline CoRoT limb-darkening coefficient $u_{\mathrm{a}}$ & $0.41 \pm 0.09$ \\
\hline CoRoT limb-darkening coefficient $u_{\mathrm{b}}$ & $0.22 \pm 0.17$ \\
\hline Spitzer limb-darkening coefficient $q_{1}$ & $0.035_{-0.020}^{+0.037}$ \\
\hline Spitzer limb-darkening coefficient $q_{2}$ & $0.39_{-0.27}^{+0.36}$ \\
\hline Spitzer limb-darkening coefficient $u_{\mathrm{a}}$ & $0.14_{-0.08}^{+0.10}$ \\
\hline Spitzer limb-darkening coefficient $u_{\mathrm{b}}$ & $0.037_{-0.11}^{+0.14}$ \\
\hline Systemic velocity $V_{\mathrm{r}}\left[\mathrm{km} \mathrm{s}^{-1}\right]$ & $19.8150 \pm 0.0017$ \\
\hline Radial-velocity jitter $s_{\mathrm{j}}\left[\mathrm{m} \mathrm{s}^{-1}\right]$ & $<3.9$ \\
\hline \multicolumn{2}{|l|}{ Transit and orbital parameters } \\
\hline Orbital period $P[\mathrm{~d}]$ & $95.272656 \pm 0.000068$ \\
\hline Transit epoch $T_{0}\left[\mathrm{BJD}_{\mathrm{TDB}}-2450000\right]^{b}$ & $5365.52723 \pm 0.00037$ \\
\hline Transit duration $T_{14}[\mathrm{~d}]$ & $0.3445_{-0.0018}^{+0.0021}$ \\
\hline CoRoT bandpass radius ratio $R_{\mathrm{p}} / R_{*}{ }^{c}$ & $0.11402_{-0.00085}^{+0.00095}$ \\
\hline Spitzer bandpass radius ratio $R_{\mathrm{p}} / R_{*}{ }^{c}$ & $0.11284_{-0.00092}^{+0.00086}$ \\
\hline Inclination $i[\mathrm{deg}]$ & $89.900_{-0.084}^{+0.066}$ \\
\hline$a / R_{*}$ & $89.9 \pm 5.9$ \\
\hline Impact parameter $b$ & $0.16_{-0.09}^{+0.11}$ \\
\hline$\sqrt{e} \cos \omega$ & $0.29_{-0.08}^{+0.06}$ \\
\hline$\sqrt{e} \sin \omega$ & $0.19_{-0.16}^{+0.00}$ \\
\hline Orbital eccentricity $e$ & $0.133_{-0.037}^{+0.042}$ \\
\hline Argument of periastron $\omega[\mathrm{deg}]$ & $41_{-24}^{+31}$ \\
\hline Radial-velocity semi-amplitude $K\left[\mathrm{~m} \mathrm{~s}^{-1}\right]$ & $39.0 \pm 2.4$ \\
\hline \multicolumn{2}{|l|}{ Planetary parameters } \\
\hline Planet mass $M_{\mathrm{p}}\left[M_{\mathrm{Jup}}\right]$ & $0.84 \pm 0.05$ \\
\hline Planet radius $R_{\mathrm{p}}\left[R_{\mathrm{Jup}}\right]$ & $1.066_{-0.063}^{+0.075}$ \\
\hline Planet density $\rho_{\mathrm{p}}\left[\mathrm{g} \mathrm{cm}^{-3}\right]$ & $0.86_{-0.16}^{+0.18}$ \\
\hline Planet surface gravity $\log g_{\mathrm{p}}[\mathrm{cgs}]$ & $3.26 \pm 0.06$ \\
\hline Orbital semimajor axis $a$ [AU] & $0.4021 \pm 0.0054$ \\
\hline Equilibrium temperature $T_{\text {eq }}[\mathrm{K}]^{d}$ & $420 \pm 16$ \\
\hline
\end{tabular}

Notes. ${ }^{(a)}$ Values from D10. ${ }^{(b)}$ In the planet-reference frame. ${ }^{(c)}$ Two dilution factors were fitted along with the radius ratios for the transits observed by Spitzer in 2010 and CoRoT in 2011 (see text for more details); their values were found to be $13.2 \pm 1.5 \%$ (Spitzer) and <0.4\% (CoRoT). ${ }^{(d)}$ Black-body equilibrium temperature assuming a null Bond albedo and uniform heat redistribution to the nightside.

of the small eccentricity of CoRoT-9b increases from 2.75 (D10) to $3.6 \sigma$, where $e=0.133_{-0.037}^{+0.042}$. To investigate whether the CoRoT-9b small eccentricity is bona fide or might be spurious, we used a Bayesian model comparison to compute the relative probabilities of the eccentric versus circular orbit models by fitting a Keplerian model to the HARPS RVs with Gaussian priors imposed on the photometric transit time and orbital period (Table 2). For this purpose, we computed the Bayesian evidence 
for both the circular and eccentric models with the Perrakis et al. (2014) method and its implementation as described in Díaz et al. (2016). We found $B_{\text {ecc,circ }}=124 \pm 8$ in favour of the eccentric model, which means that the eccentric model is $\sim 124$ times more likely than the circular model. According to Kass \& Raftery (1995), this value of Bayes factor largely exceeds the threshold $(B=20)$ of strong evidence for a more complex model, in our case for the eccentric model with respect to the circular model. This is a significant improvement with respect to the old HARPS data published by D10 because those data only yield $B_{\text {ecc,circ }}=4.3 \pm 0.4$, which is below the aforementioned threshold to claim a significant eccentricity.

\subsection{Is CoRoT-9b alone?}

As shown in Fig. 2 (lower panels), the residuals of the RVs appear flat, not showing any significant variation attributable to the presence of an additional planetary companion. From these residuals spanning almost five years, we computed the detection limits given the sampling and precision of the HARPS RVs. To this end, we injected into the residuals artificial Keplerian signals of a hypothetical companion by varying its minimum mass and orbital period with logarithmic grids from $1 M_{\oplus}$ to $5 M_{\text {Jup }}$ and from $1 \mathrm{~d}$ to $5 \mathrm{yr}$, respectively. For a given minimum mass and orbital period, we generated 500 different realisations of Keplerian models with randomly chosen values of periastron time, argument of periastron, and eccentricity; the maximum allowed eccentricity for each combination of mass and period of the simulated planet was determined from the semi-empirical stability criteria of Giuppone et al. (2013) in the most conservative case, that is by assuming that the orbit of CoRoT-9b and its hypothetical companion are anti-aligned. We carried out simulations by considering only a circular orbit for the second planet as well. Then we made use of both the F-test and the $\chi^{2}$ statistics to exclude planetary companions of a given mass and period that would induce RV variations that are incompatible at $99 \%$ confidence level with the observed RV residuals (Fig. 2). In such a way, we derived the upper limits on the minimum mass of a putative second planet as a function of orbital period. These are shown in Fig. 3 for circular (black area) and eccentric (grey contours) orbits. The region around the orbital period of CoRoT-9b is empty because it is dynamically unstable for the planetary minimum masses that are detectable with the gathered HARPS RVs. The peaks at $P \sim 1$ and 2 yr are due to the temporal sampling of the RV measurements that is inevitably affected by the object's visibility. From these detection limits, we are able to rule out the presence of massive companions of CoRoT-9b, specifically companions with $M_{\mathrm{p}} \sin i \gtrsim 0.25,1.2$, and $1.4 M_{\mathrm{Jup}}$ and $P \sim 10 \mathrm{~d}, 3 \mathrm{yr}$, and $5 \mathrm{yr}$, respectively.

The TTVs show no significant variations from a linear ephemeris either (see Fig. 4 and Table 3). This also suggests the absence of a strong perturber, even though only four transit epochs could be determined.

By considering the r.m.s. of the CoRoT light curve (Sect. 2.1) and $S / N=10$ as the transit detection threshold (Bonomo et al. 2012), we can exclude the presence of inner (coplanar) transiting planets in the system with $R_{\mathrm{p}} \gtrsim 1.3,2.0,2.3$, and $2.6 R_{\oplus}$ and orbital periods $P=1,10,25$, and $50 \mathrm{~d}$, respectively.

\section{Origin of the non-circular orbit of Corot-9b}

We now consider the question of the evolution of CoRoT-9b. Given its clearly detected non-zero eccentricity, we place constraints on the dynamical history of the CoRoT-9 system. We
Table 3. Times of CoRoT-9b mid-transits.

\begin{tabular}{lll}
\hline \hline $\begin{array}{l}\text { Time } \\
{\left[\mathrm{BJD}_{\mathrm{TDB}}-2450000\right]}\end{array}$ & $\begin{array}{l}\text { Uncertainty } \\
\text { [days] }\end{array}$ & Instrument \\
\hline 4603.34577 & 0.00061 & CoRoT \\
4698.6164 & 0.0019 & CoRoT \\
5746.61705 & 0.00074 & CoRoT \\
5365.52764 & 0.00087 & Spitzer \\
5746.61825 & 0.00093 & Spitzer \\
\hline
\end{tabular}

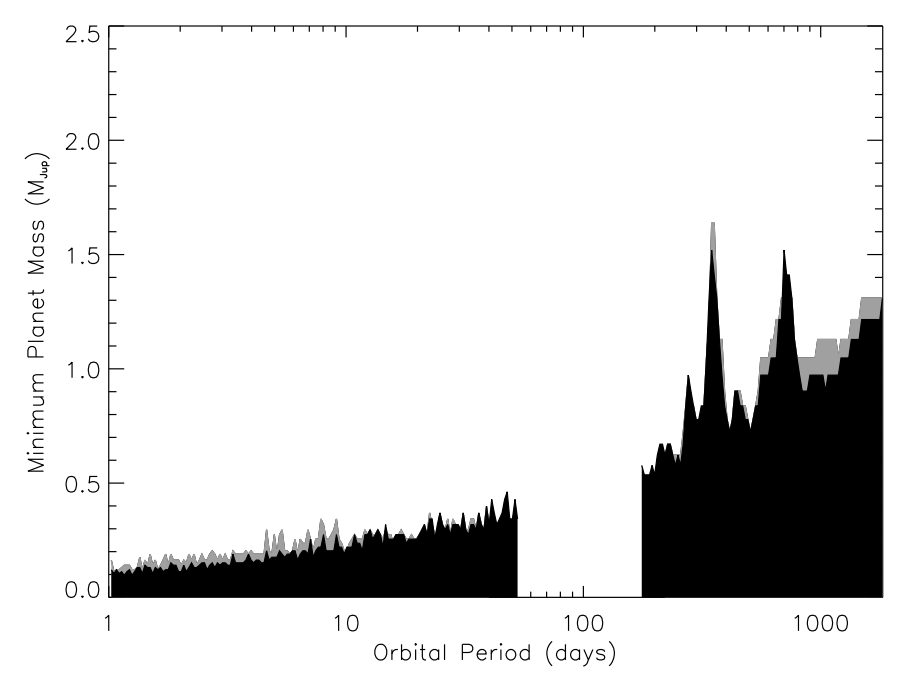

Fig. 3. Upper limits on the minimum mass of a possible planetary companion of CoRoT-9b with $99 \%$ confidence level as a function of orbital period up to 5 years. Black and shaded areas refer to circular and eccentric orbits of the hypothetical companion; the maximum allowed eccentricity for each period was derived from dynamical stability criteria (see text for more details). The empty region around the orbital period of CoRoT-9b $(P=95.27 \mathrm{~d})$ is dynamically unstable for the minimum planetary masses detectable with our HARPS RV data.

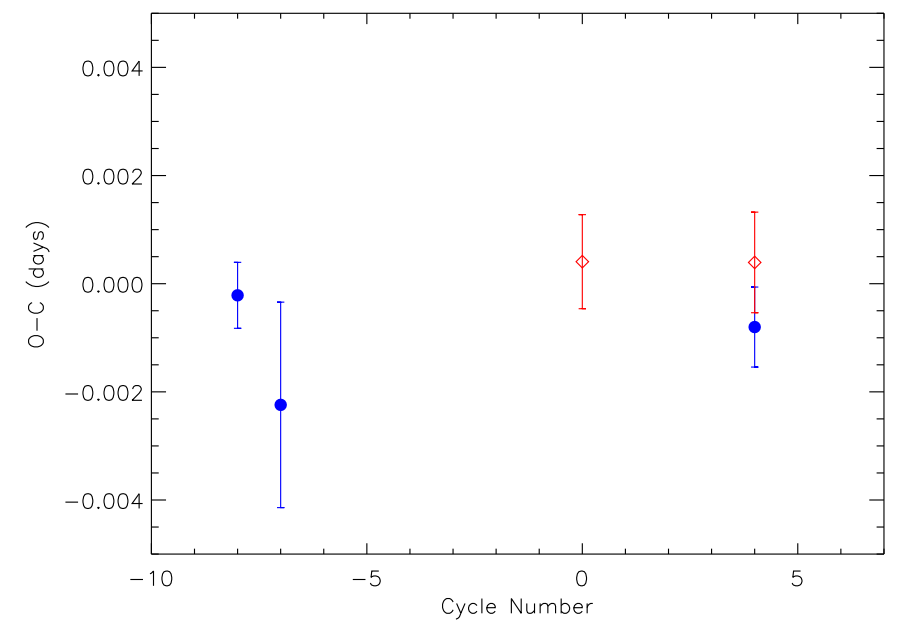

Fig. 4. Residuals of the mid-transit epochs of CoRoT-9b vs. the linear ephemeris reported in Table 2. Blue filled circles refer to the transits observed by CoRoT; empty red diamonds indicate the two Spitzer transits. The value with the largest error bar comes from a partial CoRoT transit.

first discuss a broad range of potential formation models, culminating with what we consider to be the most likely candidate: planet-planet scattering. Next we present a suite of scattering simulations to reproduce the orbit of CoRoT-9b. Finally, we construct a plausible formation scenario for the system. Our results 
provide motivation to measure the sky-projected obliquity of the CoRoT-9 system.

\subsection{Possible evolutionary scenarios for the CoRoT-9 system}

There exist a number of mechanisms that could explain the origin of the CoRoT-9 system.

1. In situ formation of CoRoT-9b from local material at $\sim 0.4 \mathrm{AU}$. It is possible that there was enough material in the disk for the planet to grow a core of several Earth masses and to accrete gas from the disk (e.g. Ikoma et al. 2001; Hubickyj et al. 2005; Raymond et al. 2008; Batygin et al. 2016). However, if the planet formed in situ, it is hard to understand why only one planet should have formed. And even if there exist additional planets that are too small to detect, why would CoRoT-9b have an eccentric orbit? Additional low-mass planets cannot pump the eccentricity of CoRoT-9b to its observed value. Isolated in situ accretion is implausible.

2. Formation of CoRoT-9b farther from the star followed by gas-driven inward migration. Migration is indeed a likely - and unavoidable - consequence of planetdisk interaction (Goldreich \& Tremaine 1980; Ward 1986; Lin \& Papaloizou 1986; Papaloizou \& Terquem 2006). Migration is usually directed inward and can plausibly explain the origin of close-in planets of a wide range of masses (Kley \& Nelson 2012; Baruteau et al. 2014). However, simulations show that orbital migration of a single planet universally lowers the orbital eccentricity of a planet (Tanaka \& Ward 2004; Cresswell \& Nelson 2008; Bitsch \& Kley 2010) except in the extreme case of a very massive planet $\left(M_{\mathrm{p}} \sim 5-10 M_{\mathrm{Jup}}\right)$ in a very massive disk (Papaloizou et al. 2001; Kley \& Dirksen 2006; Dunhill et al. 2013). The non-zero eccentricity of CoRoT-9b appears to rule out a solitary migration scenario.

3. Inward migration of CoRoT-9b driven by secular forcing from a more distant giant planet. Petrovich \& Tremaine (2016) proposed that WJs are driven inward by a combination of secular forcing from a distant companion and tidal dissipation (see also Dawson \& Chiang 2014). In this model, the eccentricity of the inner planet is periodically driven to such high values - and its perihelion distance to such low values - that tidal dissipation shrinks the orbit of the planet. The inner planet is thus driven inward in periodic bursts. However, this model requires the presence of a second planet on a more distant, very eccentric orbit. There is no hint of such a distant perturber (see Sect. 4.2). While constraints on additional planets in the CoRoT-9 system cannot completely rule out this model, it is worth noting that the model struggles to produce WJs with modest $(e \lesssim 0.2)$ eccentricities. This scenario appears unlikely to explain the CoRoT-9 system.

4. CoRoT-9b as the survivor of a dynamical instability. The planet-planet scattering model can explain the broad eccentricity distribution of observed giant exoplanets (e.g. Adams \& Laughlin 2003; Chatterjee et al. 2008; Jurić \& Tremaine 2008; Ford \& Rasio 2008; Raymond et al. 2010). This model proposes that the observed planets are the survivors of violent dynamical instabilities in which multiple planets underwent close gravitational encounters. During these gravitational scattering events, one or more planets are lost, usually by ejection into interstellar space (although their numbers are too low to explain the abundance of freefloating gas giants; Veras \& Raymond 2012). The CoRoT9 system could have formed with one or more additional planets whose orbits became unstable. Given the proximity of CoRoT-9b to the star, the planets may have migrated inward and then become unstable when the gas disk dissipated (see e.g. Ogihara \& Ida 2009; Cossou et al. 2014).

The planet-planet scattering mechanism operates when the gravitational kick of a planet is strong enough that scattering dominates over accretion. This is often quantified with the so-called Safronov number $\Theta$ (Safronov \& Zvjagina 1969), which is defined as the ratio of the escape speed from the surface of a planet to the escape speed from the star, or

$\Theta^{2}=\left(\frac{G M_{\mathrm{p}}}{R_{\mathrm{p}}}\right)\left(\frac{a}{G M_{\star}}\right)=\left(\frac{M_{\mathrm{p}}}{M_{\star}}\right)\left(\frac{a}{R_{\mathrm{p}}}\right)$,

where $M_{\mathrm{p}}$ and $M_{\star}$ are the planetary and stellar masses, respectively, $R_{\mathrm{p}}$ is the planet's radius and $a$ is the orbital radius. Giant exoplanets with larger $\Theta$ are observed to have higher eccentricities (Ford et al. 2001; Ford \& Rasio 2008). For the case of CoRoT- $9 b, \Theta=0.66$, that is, close to unity. This puts the planet right at the boundary between the scattering and accretionary regimes. While a scattering origin appears plausible to explain the origin of the non-zero eccentricity of CoRoT-9b, it is worth checking with numerical simulations.

\subsection{Scattering simulations to explain the orbit of CoRoT-9b}

We ran a suite of numerical simulations of planet-planet scattering. The goal of these simulations was to test whether the orbit of CoRoT-9b can be reproduced by planet scattering. In this context, CoRoT-9b would represent the survivor of a dynamical instability that removed another planet from the system, likely by dynamical ejection. For simplicity we only included one additional planet.

Each simulation contained a star with the properties of CoRoT-9 orbited by two planets: a CoRoT-9b analogue with the actual mass of the planet, and a second planet. CoRoT-9b analogues were initially placed at $0.46 \mathrm{AU}$ on circular orbits. This orbital radius was chosen so that, after ejecting a $50 M_{\oplus}$ companion, CoRoT-9b would end up on its actual orbit. This initial radius would shift by up to $\sim 0.1$ AU for CoRoT-9b to end up on its actual orbit for the range of extra planet masses considered. While a different initial radius for CoRoT-9b would modestly change its starting Safronov number, this would not affect the outcome of scattering. In fact, we show below that, while changing the Safronov number of CoRoT-9b impacts the branching ratios of different outcomes (i.e. a lower $\Theta^{2}$ implies a higher rate of collisions), it does not change the outcomes themselves (i.e. the final eccentricity of CoRoT-9b is not sensitive to the value of $\Theta^{2}$ ). The extra planet was placed on an exterior orbit within $2 \%$ of the $3: 4$ or $4: 5$ mean motion resonances ${ }^{5}$. The reason for this was to remain consistent with a migration origin for the planets, which generally implies resonant capture (e.g. Kley \& Nelson 2012), while being dynamically unstable (Marchal \& Bozis 1982; Gladman 1993). The orbits of the planets were given a randomly chosen small $\left(<1^{\circ}\right)$ initial mutual inclination.

We tested two parameters: the mass of the second planet and the physical density of both planets. We considered extra planet masses $M_{\text {extra }}=25,50,75,100,200$ and $267 M_{\oplus}$ (the mass of CoRoT-9b is $267 M_{\oplus}$ ). For an extra planet mass of

\footnotetext{
For one set of simulations we also tested placing the extra planet on a near-resonant orbit interior to the CoRoT-9b analogues and found no
} measurable difference in outcome. 


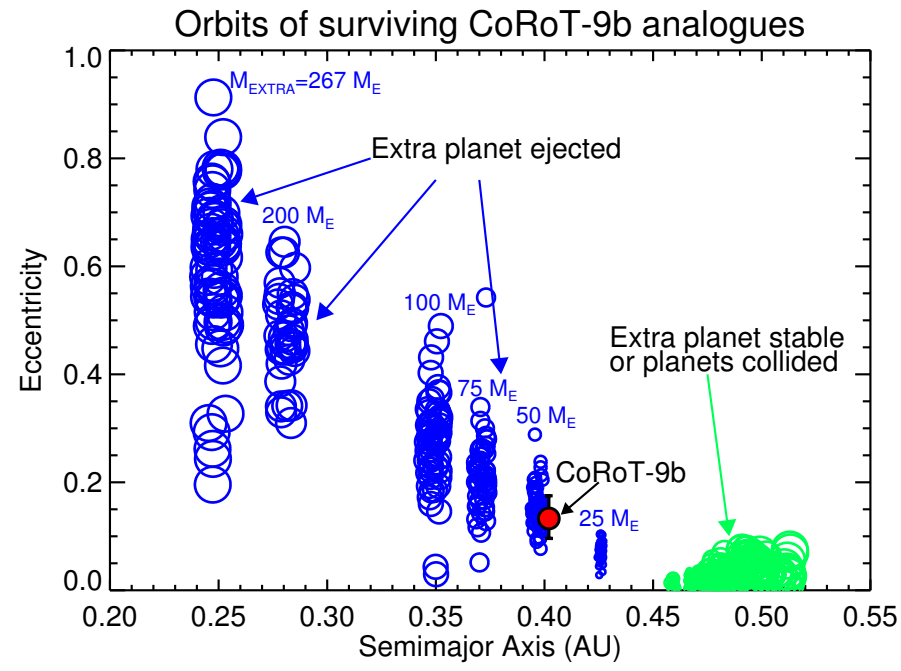

Fig. 5. Orbital semimajor axes and eccentricities of surviving CoRoT-9b analogues from our numerical simulations. The blue planets are those that remained after the second planet was ejected; each vertical band corresponds to a specific mass for the extra planet. The green circles are simulations in which either there was no instability or the two planets collided. The measured orbit of CoRoT-9b is shown with the red symbol; the error bars are $1 \sigma$.

$50 M_{\oplus}$ we also tested physical densities for the planets of 0.5 , 1.0 , and $2.0 \mathrm{~g} \mathrm{~cm}^{-3}$. In all other simulations the densities of both planets were fixed at $1.0 \mathrm{~g} \mathrm{~cm}^{-3}$; even though this value is consistent with the measured density of CoRoT-9b within $1 \sigma$ $\left(0.86_{-0.16}^{+0.18} \mathrm{~g} \mathrm{~cm}^{-3}\right)$, we show below that the density has no effect on the outcome. For each set we ran 100 simulations.

Each simulation was integrated for 10 million years or until the system became unstable and one planet was removed. We used the Mercury hybrid integrator (Chambers 1999) with a timestep of 0.1 days. This timestep was small enough to accurately resolve orbits that collided with the star, which were assumed in the calculations to have radii of $0.01 \mathrm{AU}$ (see Appendix A of Raymond et al. 2011, for representative numerical tests). Planets were removed from the system if their orbital radii reached $1000 \mathrm{AU}$. When this happened, collisions between the planets were treated as inelastic mergers.

Figure 5 shows the orbits of CoRoT-9b analogues at the end of the simulations. The small clump at 0.46 AU represents systems that remained stable; in those cases CoRoT-9b remained on a circular orbit. In simulations in which the two planets collided, CoRoT-9b analogues are at slightly larger orbital radii (0.47-0.52 AU) and with small eccentricities (typically $e \lesssim 0.05)$. A collision between CoRoT-9b and an extra planet is clearly inconsistent with the measured eccentricity of CoRoT-9b.

When the orbits of the planets become unstable and the extra planet is ejected, CoRoT-9b analogues are shifted inward from their initial orbits. The outcomes are radially segregated by the mass of the extra planet because of the mass dependence of the orbital energy exchanged when the extra planet was ejected. In simple terms, CoRoT-9b feels a mass-dependent recoil from ejecting the extra planet. Thus, each vertical "stripe" in Fig. 5 represents the outcome of a set of simulations with a specified extra planet mass. It is important to note that these simulations were designed to reproduce the eccentricity of CoRoT-9b, not its semimajor axis. Thus, even though the simulations with $M_{\text {extra }}=$ $50 M_{\oplus}$ are the closest match in semimajor axis, other sets of simulations could easily match the semimajor axis of CoRoT-9b; for

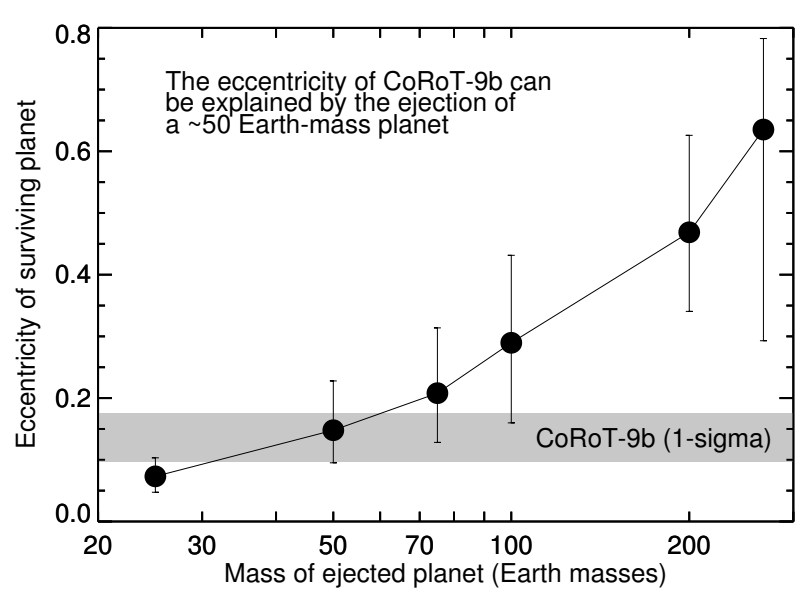

Fig. 6. Eccentricity of surviving planets as a function of the mass of the ejected planet. The large points show the median for each set and the error bars show the 5th-95th percentile of outcomes. The shaded area shows the $\pm 1 \sigma$ range of the measured eccentricity of CoRoT-9b.

example, the simulations with $M_{\text {extra }}=75 M_{\oplus}$ would match if CoRoT-9b had started at $\sim 0.49$ AU instead of 0.46 AU.

The eccentricities of surviving CoRoT-9b analogues correlate with the mass of the ejected planet. The ejection of a $25 M_{\oplus}$ planet only excites CoRoT-9b analogues to an eccentricity of $\sim 0.05$, whereas ejecting a planet comparable in mass can excite the eccentricities of the planets up to 0.9. Nonetheless, there is a broad range in eccentricities of CoRoT-9b analogues for each set of simulations with a specified extra planet mass. This is because the final eccentricity depends on the details of the last close encounters between the planets, whose alignment is stochastic in nature.

Figure 6 shows the eccentricity of CoRoT-9b analogues after ejecting an extra planet. It is clear that the set of simulations with $M_{\text {extra }}=50 M_{\oplus}$ most readily produces CoRoT-9b analogues with the measured eccentricity. However, there is a small tail of outcomes with $M_{\text {extra }}=25 M_{\oplus}$ that overlaps with the lower allowed values for CoRoT-9b. At larger masses, a significant fraction of simulations with $M_{\text {extra }}=75 M_{\oplus}$ overlap with the higher allowed values, and even some simulations with $M_{\text {extra }}=100 M_{\oplus}$ are allowed by observations.

Planet-planet scattering also excites the planetary orbital inclinations (Jurić \& Tremaine 2008; Chatterjee et al. 2008; Raymond et al. 2010). Indeed, scattering of planets to extremely high eccentricities followed by tidal dissipation has been proposed as a mechanism to produce hot Jupiters whose orbits are misaligned with the stellar equator (Nagasawa et al. 2008; Beaugé \& Nesvorný 2012).

Figure 7 shows the inclinations of surviving CoRoT-9b analogues. None of the planets that match the eccentricity of CoRoT-9b have inclinations larger than $3^{\circ}$. Inclinations above $10^{\circ}$ were only produced in the most energetic scattering events, which also stranded CoRoT-9b analogues on orbits much more eccentric than the real planet.

Our simulations thus predict that the orbit of CoRoT-9b should be in the same plane as it started, to within a few degrees. If we assume that plane to have been aligned with the stellar equator, then this naturally predicts that Rossiter-McLaughlin measurements should find a low stellar obliquity for CoRoT-9, i.e. an alignment between the planetary orbital plane and the stellar equator.

But what would it mean if Rossiter-McLaughlin measurements found a non-zero stellar obliquity? Given the arguments 


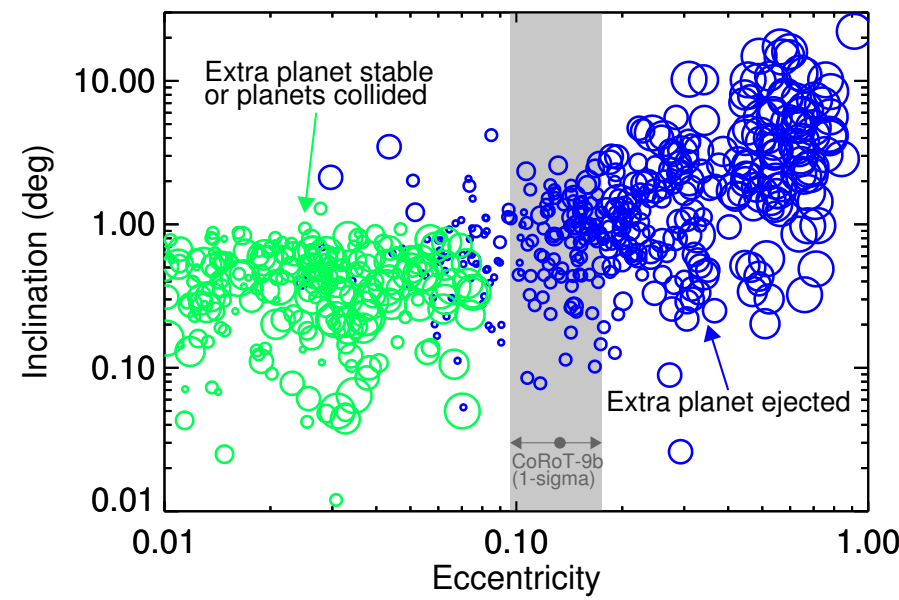

Fig. 7. Orbital inclination (zero degrees correspond to the orbital plane prior to scattering phase) vs. eccentricity for CoRoT-9b analogues at the end of our simulations. As in Fig. 5, blue circles represent planets that survived an instability whereas green circles represent planets that either remained on stable orbits or collided with the extra planet (recall that the initial inclination was randomly chosen between zero and $1^{\circ}$ ). The size of the circle correlates with the mass of the extra planet. The shaded region represents the $1 \sigma$ allowed eccentricities for CoRoT-9b.

presented above, we think that planet scattering is by far the most likely origin for the eccentric orbit of CoRoT-9b. Assuming that scattering indeed took place, a measured non-zero stellar obliquity would imply that the planetary orbital plane was already misaligned prior to the scattering phase. This would be strong indirect evidence for misalignment of the protoplanetary disk of CoRoT-9. Some candidate mechanisms to tilt disks are chaotic star formation (Bate et al. 2010), magnetic star-disk interactions (Lai et al. 2011), and perturbations from a distant stellar binary (Batygin 2012; Batygin \& Adams 2013; Lai 2014). Concerning the latter, even temporary binarity during the embedded cluster phase may tilt the disk.

Finally, we also tested the effect of the planetary density $\rho$ on the outcome of scattering simulations. This could be important because the density is linked to the radius of the planet and therefore its escape speed, and thus the Safronov number $\Theta$.

In the simulations presented above we assumed that both the CoRoT-9b analogues and the extra planet had densities of $\rho=$ $1 \mathrm{~g} \mathrm{~cm}^{-3}$. We ran three sets of 100 simulations each with $M_{\text {extra }}=$ $50 M_{\oplus}$ and varying the density (of both planets) between 0.5 and $2.0 \mathrm{~g} \mathrm{~cm}^{-3}$. This corresponded to a range in $\Theta^{2}$ between 0.55 and 0.87 . All other aspects of the simulations were as above.

Figure 8 shows the surviving planets in the three sets of simulations with different densities. At a glance the outcomes of the simulations appear similar. Indeed, Kolmogorov-Smirnov tests show that, after ejecting the extra planet, the distribution of eccentricities of CoRoT-9b analogues in all three sets are consistent with having been drawn from the same distribution.

Yet the branching ratios between outcomes did depend on the planet density. In the set of simulations with $\rho=0.5 \mathrm{~g} \mathrm{~cm}^{-3}$, collisions between the two planets were more than twice as common (53 collisions versus 24) as in the set of simulations with $\rho=2 \mathrm{~g} \mathrm{~cm}^{-3}$. Ejections were significantly less common in the systems with low-density planets (42 ejections for $\rho=0.5 \mathrm{~g} \mathrm{~cm}^{-3}$ vs. 67 for $\rho=2.0 \mathrm{~g} \mathrm{~cm}^{-3}$ ). The simulations with $\rho=1.0 \mathrm{~g} \mathrm{~cm}^{-3}$ were intermediate in both cases.

We can conclude from this simple numerical experiment that, while the planet density affects the probability of a given outcome, it has little effect on the details of that outcome.

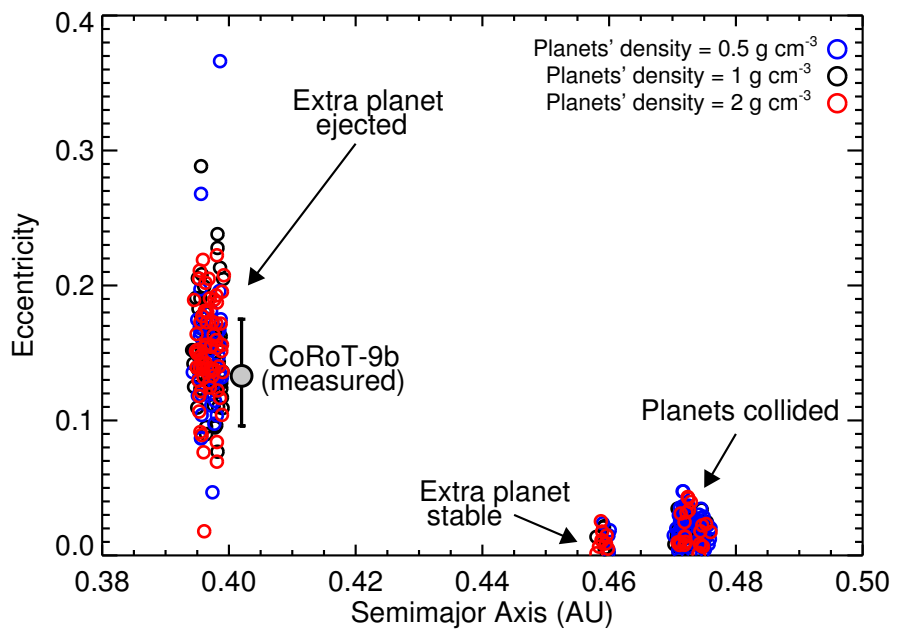

Fig. 8. Orbital eccentricity vs. semimajor axis for surviving CoRoT$9 \mathrm{~b}$ analogues in three sets of simulations that varied the densities of the planets. The blue, black, and red symbols represent simulations in which $\rho=0.5,1.0$, and $2.0 \mathrm{~g} \mathrm{~cm}^{-3}$, respectively.

\subsection{A plausible evolutionary history for CoRoT-9b}

Given the discussion above and the results of our scattering simulations, we propose the following evolutionary history for the CoRoT-9 system.

- CoRoT-9b formed relatively late in the lifetime of the protoplanetary disk, probably at a few AU, and migrated inward. Its migration stopped at $\sim 0.5 \mathrm{AU}$ because the disk was starting to photo-evaporate (Alexander \& Pascucci 2012).

- A second, $\sim 50 M_{\oplus}$ planet formed in the system. This may have been a second core that formed farther out, migrated inward, and caught up with CoRoT-9b (as in e.g. the Grand Tack model for the Solar System; Walsh et al. 2011), or perhaps a planet whose growth was accelerated by the migration of CoRoT-9b via the "snowplow" effect of migrating resonances (Zhou et al. 2005; Fogg \& Nelson 2005; Raymond et al. 2006; Mandell et al. 2007). The extra planet became locked in (or near; Adams et al. 2008) mean motion resonance with CoRoT-9b.

- The orbits of CoRoT-9b and the extra planet became unstable after the dispersal of the gaseous disk. The two planets scattered off of each other, leading to the ejection of the smaller planet. Nearby small planets were destroyed during the instability (Raymond et al. 2011, 2012). The measured eccentricity of CoRoT-9b is essentially a scar from this instability.

\section{Summary and conclusions}

Thanks to three new space-based observations of transits of CoRoT-9b with CoRoT and Spitzer and a $\sim 5-\mathrm{yr}$ RV monitoring with HARPS, we updated the CoRoT-9b physical parameters, $M_{\mathrm{p}}=0.84 \pm 0.05 M_{\mathrm{Jup}}, R_{\mathrm{p}}=1.066_{-0.063}^{+0.075} R_{\mathrm{Jup}}$, and $\rho_{\mathrm{p}}=0.86_{-0.16}^{+0.18} \mathrm{~g} \mathrm{~cm}^{-3}$, which agree well with the literature values (D10). With the new RV data, we found a higher significance for the non-zero eccentricity $e=0.133_{-0.037}^{+0.042}$ of CoRoT-9b and no evidence for additional companions. The TTVs do not deviate significantly from a linear ephemeris either, thus supporting the absence of a strong perturber, even though only four transit epochs could be determined. Inner transiting (coplanar) planets with $R_{\mathrm{p}} \gtrsim 1.3,2.0,2.3$, and $2.6 R_{\oplus}$ and orbital periods $P=1,10$, 
25, and $50 \mathrm{~d}$, respectively, can be excluded as well using CoRoT data.

We investigated different scenarios to reproduce the orbit of CoRoT-9b, assuming a lack of additional companions. We argue that in situ formation, secular interactions with an outer perturber, and single planet migration are all unlikely to reproduce the CoRoT-9 system. Instead we believe that planet-planet scattering is the most likely origin story for the system.

Using simulations of planet-planet scattering we showed that the eccentricity of CoRoT-9b is likely a residual scar from an instability that ejected a planet of roughly $50 M_{\oplus}$. Although we only performed simulations with a single additional planet, we expect that the same process could have occurred with more planets. The ejection of multiple planets with a total of $\sim 50 M_{\oplus}$ could induce a comparable eccentricity in CoRoT-9b.

Our simulations also serve to motivate future observations of the Rossiter-McLaughlin effect of CoRoT-9b, although challenging given the long transit duration of $8.3 \mathrm{~h}$ (but see e.g. Hébrard et al. 2010, for the case of HD 80606 b). While planetplanet scattering of a $\sim 50 M_{\oplus}$ planet can excite the eccentricity of CoRoT-9b to its current value, there is little associated inclination excitation. The orbital plane of CoRoT-9b should not have changed since before the instability. We thus predict a spinorbit alignment for the system. Yet perhaps the most interesting outcome would be a measured misalignment, which would be strong indirect evidence that the orbital plane of CoRoT-9b was already misaligned before the instability, perhaps indicating that the protoplanetary disk of the star was tilted at a young age.

Acknowledgements. This work is based in part on observations made with the Spitzer Space Telescope, which is operated by the Jet Propulsion Laboratory, California Institute of Technology under a contract with NASA. A.S.B. acknowledges funding from the European Union Seventh Framework programme (FP7/2007-2013) under grant agreement No. 313014 (ETAEARTH). G.H. and A.L. acknowledge the support by the CNES and the French Agence Nationale de la Recherche (ANR), under programme ANR-12-BS05-0012 "Exo-Atmos". S.N.R. and A.I. thank the Agence Nationale pour la Recherche for support via grant ANR-13-BS05-0003- 01 (project MOJO). A.I. also thanks partial financial support from FAPESP (Proc. 16/12686-2 and 16/19556-7). R.A. acknowledges the Spanish Ministry of Economy and Competitiveness (MINECO) for the financial support under the Ramón y Cajal programme RYC-2010-06519, and under the programme RETOS ESP2014-57495-C2-1-R and ESP2016-80435-C2-2R. H.D. acknowledges support by grant ESP2015-65712-C5-4-R of the Spanish Secretary of State for R\&D\&i (MINECO).

\section{References}

Adams, F. C., \& Laughlin, G. 2003, Icarus, 163, 290

Adams, F. C., Laughlin, G., \& Bloch, A. M. 2008, ApJ, 683, 1117

Aigrain, S., Pont, F., Fressin, F., et al. 2009, A\&A, 506, 425

Alexander, R. D., \& Pascucci, I. 2012, MNRAS, 422, 82

Anderson, D. R., Collier Cameron, A., Hellier, C., et al. 2011, ApJ, 726, L19

Ballerini, P., Micela, G., Lanza, A. F., \& Pagano, I. 2012, A\&A, 539, A140

Baranne, A., Queloz, D., Mayor, M., et al. 1996, A\&AS, 119, 373

Barros, S. C. C., Almenara, J. M., Deleuil, M., et al. 2014, A\&A, 569, A74

Baruteau, C., Crida, A., Paardekooper, S.-J., et al. 2014, Protostars and Planets VI, 667

Bate, M. R., Lodato, G., \& Pringle, J. E. 2010, MNRAS, 401, 1505

Batygin, K. 2012, Nature, 491, 418

Batygin, K., \& Adams, F. C. 2013, ApJ, 778, 169

Batygin, K., Bodenheimer, P. H., \& Laughlin, G. P. 2016, ApJ, 829, 114

Beaugé, C., \& Nesvorný, D. 2012, ApJ, 751, 119

Bitsch, B., \& Kley, W. 2010, A\&A, 523, A30

Bonomo, A. S., Aigrain, S., Bordé, P., \& Lanza, A. F. 2009, A\&A, 495, 647

Bonomo, A. S., Santerne, A., Alonso, R., et al. 2010, A\&A, 520, A65

Bonomo, A. S., Chabaud, P. Y., Deleuil, M., et al. 2012, A\&A, 547, A110

Bonomo, A. S., Sozzetti, A., Lovis, C., et al. 2014, A\&A, 572, A2

Bonomo, A. S., Sozzetti, A., Santerne, A., et al. 2015, A\&A, 575, A85
Bonomo, A. S., Desidera, S., Benatti, S., et al. 2017, A\&A, 602, A107 Borsato, L., Marzari, F., Nascimbeni, V., et al. 2014, A\&A, 571, A38 Bruno, G., Almenara, J.-M., Barros, S. C. C., et al. 2015, A\&A, 573, A124 Bryan, M. L., Knutson, H. A., Howard, A. W., et al. 2016, ApJ, 821, 89 Chambers, J. E. 1999, MNRAS, 304, 793

Chatterjee, S., Ford, E. B., Matsumura, S., \& Rasio, F. A. 2008, ApJ, 686, 580 Claret, A., \& Bloemen, S. 2011, A\&A, 529, A75

Cossou, C., Raymond, S. N., Hersant, F., \& Pierens, A. 2014, A\&A, 569, A56 Cresswell, P., \& Nelson, R. P. 2008, A\&A, 482, 677

Dawson, R. I., \& Chiang, E. 2014, Science, 346, 212

Dawson, R. I., Johnson, J. A., Morton, T. D., et al. 2012, ApJ, 761, 163

Deeg, H. J., Gillon, M., Shporer, A., et al. 2009, A\&A, 506, 343

Deeg, H. J., Moutou, C., Erikson, A., et al. 2010, Nature, 464, 384

Demarque, P., Woo, J.-H., Kim, Y.-C., \& Yi, S. K. 2004, ApJS, 155, 667

Demory, B.-O., \& Seager, S. 2011, ApJS, 197, 12

Díaz, R. F., Ségransan, D., Udry, S., et al. 2016, A\&A, 585, A134

Dunhill, A. C., Alexander, R. D., \& Armitage, P. J. 2013, MNRAS, 428, 3072

Eastman, J., Siverd, R., \& Gaudi, B. S. 2010, PASP, 122, 935

Eastman, J., Gaudi, B. S., \& Agol, E. 2013, PASP, 125, 83

Fazio, G. G., Hora, J. L., Allen, L. E., et al. 2004, ApJS, 154, 10

Fogg, M. J., \& Nelson, R. P. 2005, A\&A, 441, 791

Ford, E. B., \& Rasio, F. A. 2008, ApJ, 686, 621

Ford, E. B., Havlickova, M., \& Rasio, F. A. 2001, Icarus, 150, 303

Giuppone, C. A., Morais, M. H. M., \& Correia, A. C. M. 2013, MNRAS, 436, 3547

Gladman, B. 1993, Icarus, 106, 247

Goldreich, P., \& Tremaine, S. 1980, ApJ, 241, 425

Gregory, P. C. 2005, ApJ, 631, 1198

Hamers, A. S., Antonini, F., Lithwick, Y., Perets, H. B., \& Portegies Zwart, S. F. 2017, MNRAS, 464, 688

Hébrard, G., Désert, J.-M., Díaz, R. F., et al. 2010, A\&A, 516, A95

Huang, C., Wu, Y., \& Triaud, A. H. M. J. 2016, ApJ, 825, 98

Hubickyj, O., Bodenheimer, P., \& Lissauer, J. J. 2005, Icarus, 179, 415

Ikoma, M., Emori, H., \& Nakazawa, K. 2001, ApJ, 553, 999

Izidoro, A., Raymond, S. N., Morbidelli, A., Hersant, F., \& Pierens, A. 2015, ApJ, 800, L22

Jurić, M., \& Tremaine, S. 2008, ApJ, 686, 603

Kass, R. E., \& Raftery, A. E. 1995, J. Am. Stat. Assoc., 90, 773

Kipping, D. M. 2013, MNRAS, 435, 2152

Kley, W., \& Dirksen, G. 2006, A\&A, 447, 369

Kley, W., \& Nelson, R. P. 2012, ARA\&A, 50, 211

Lai, D. 2014, MNRAS, 440, 3532

Lai, D., Foucart, F., \& Lin, D. N. C. 2011, MNRAS, 412, 2790

Lambrechts, M., Johansen, A., \& Morbidelli, A. 2014, A\&A, 572, A35

Lecavelier des Etangs, A., Hébrard, G., Blandin, S., et al. 2017, A\&A, in press DOI: $10.1051 / 0004-6361 / 201730554$

Lin, D. N. C., \& Papaloizou, J. 1986, ApJ, 309, 846

Lin, D. N. C., Bodenheimer, P., \& Richardson, D. C. 1996, Nature, 380, 606

Llebaria, A., \& Guterman, P. 2006, in The CoRoT Mission Pre-Launch

Status - Stellar Seismology and Planet Finding, eds. M. Fridlund, A. Baglin,

J. Lochard, \& L. Conroy, ESA SP, 1306, 293

Mandel, K., \& Agol, E. 2002, ApJ, 580, L171

Mandell, A. M., Raymond, S. N., \& Sigurdsson, S. 2007, ApJ, 660, 823

Marchal, C., \& Bozis, G. 1982, Cel. Mech., 26, 311

Marzari, F., Baruteau, C., \& Scholl, H. 2010, A\&A, 514, L4

Mayor, M., Pepe, F., Queloz, D., et al. 2003, The Messenger, 114, 20

Mordasini, C., Alibert, Y., Benz, W., Klahr, H., \& Henning, T. 2012, A\&A, 541, A97

Nagasawa, M., Ida, S., \& Bessho, T. 2008, ApJ, 678, 498

Ogihara, M., \& Ida, S. 2009, ApJ, 699, 824

Papaloizou, J. C. B., \& Terquem, C. 2006, Rep. Prog. Phys., 69, 119

Papaloizou, J. C. B., Nelson, R. P., \& Masset, F. 2001, A\&A, 366, 263

Pepe, F., Mayor, M., Galland, F., et al. 2002, A\&A, 388, 632

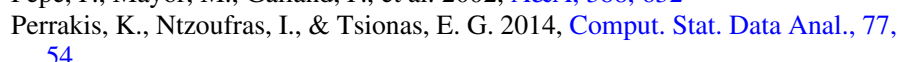

Petrovich, C., \& Tremaine, S. 2016, ApJ, 829, 132

Petrovich, C., Tremaine, S., \& Rafikov, R. 2014, ApJ, 786, 101

Rasio, F. A., \& Ford, E. B. 1996, Science, 274, 954

Raymond, S. N., Mandell, A. M., \& Sigurdsson, S. 2006, Science, 313, 1413

Raymond, S. N., Barnes, R., \& Mandell, A. M. 2008, MNRAS, 384, 663

Raymond, S. N., Armitage, P. J., \& Gorelick, N. 2010, ApJ, 711, 772

Raymond, S. N., Armitage, P. J., Moro-Martín, A., et al. 2011, A\&A, 530, A62

Raymond, S. N., Armitage, P. J., Moro-Martín, A., et al. 2012, A\&A, 541, A11

Safronov, V. S., \& Zvjagina, E. V. 1969, Icarus, 10, 109

Santerne, A., Díaz, R. F., Moutou, C., et al. 2012, A\&A, 545, A76

Santerne, A., Moutou, C., Tsantaki, M., et al. 2016, A\&A, 587, A64

Schneider, J., Dedieu, C., Le Sidaner, P., Savalle, R., \& Zolotukhin, I. 2011, A\&A, 532, A79 
Shporer, A., O’Rourke, J. G., Knutson, H. A., et al. 2014, ApJ, 788, 92 Sozzetti, A., Torres, G., Charbonneau, D., et al. 2007, ApJ, 664, 1190

Sozzetti, A., Bonomo, A. S., Biazzo, K., et al. 2015, A\&A, 575, L15

Tanaka, H., \& Ward, W. R. 2004, ApJ, 602, 388

ter Braak, C. J. F. 2006, Stat. Comput., 16, 239

Veras, D., \& Raymond, S. N. 2012, MNRAS, 421, L117

Walsh, K. J., Morbidelli, A., Raymond, S. N., O’Brien, D. P., \& Mandell, A. M. 2011, Nature, 475, 206

Ward, W. R. 1986, Icarus, 67, 164

Wu, Y., \& Lithwick, Y. 2011, ApJ, 735, 109

Zhou, J.-L., Aarseth, S. J., Lin, D. N. C., \& Nagasawa, M. 2005, ApJ, 631, L85

1 INAF-Osservatorio Astrofisico di Torino, via Osservatorio 20, 10025 Pino Torinese, Italy

e-mail: bonomo@oato.inaf.it

2 Institut d'Astrophysique de Paris, CNRS, UMR 7095 \& Sorbonne Universités, UPMC Paris 6, 98 bis bd Arago, 75014 Paris, France

3 Observatoire de Haute Provence, Université Aix-Marseille \& CNRS, 04870 St. Michel l'Observatoire, France

${ }^{4}$ Laboratoire d'Astrophysique de Bordeaux, Université de Bordeaux, CNRS, B18N, Allée Geoffroy Saint-Hilaire, 33615 Pessac, France

5 Observatoire de l'Université de Genève, 51 chemin des Maillettes, 1290 Sauverny, Switzerland

${ }^{6}$ Department of Physics, Denys Wilkinson Building Keble Road, Oxford, OX1 3RH, UK

7 Instituto de Astrofisica de Canarias, 38205 La Laguna, Tenerife, Spain

8 Universidad de La Laguna, Dept. de Astrofísica, 38200 La Laguna, Tenerife, Spain

9 Institute of Planetary Research, German Aerospace Center, Rutherfordstrasse 2, 12489 Berlin, Germany
10 Institut d'Astrophysique Spatiale, UMR 8617, CNRS - Université de Paris-Sud - Université Paris-Saclay - Bât. 121, 91405 Orsay, France

11 Aix Marseille Université, CNRS, LAM (Laboratoire d'Astrophysique de Marseille) UMR 7326, 13388 Marseille, France

12 Universidad de Buenos Aires, Facultad de Ciencias Exactas y Naturales, Buenos Aires, Argentina

13 CONICET - Universidad de Buenos Aires, Instituto de Astronomía y Física del Espacio (IAFE), Buenos Aires, Argentina

14 Leiden Observatory, University of Leiden, PO Box 9513, 2300 RA, Leiden, The Netherlands

15 Department of Earth and Space Sciences, Chalmers University of Technology, Onsala Space Observatory, 43992 Onsala, Swden

16 Dipartimento di Fisica, Università di Torino, via P. Giuria 1, 10125 Torino, Italy

17 Thüringer Landessternwarte, Sternwarte 5, Tautenburg 5, 07778 Tautenburg, Germany

18 Observatoire de la Côte d'Azur, Laboratoire Cassiopée, BP 4229, 06304 Nice Cedex 4, France

19 UNESP, Univ. Estadual Paulista - Grupo de Dinâmica Orbital \& Planetologia, Guaratinguetá, CEP 12.516-410, São Paulo, Brazil

20 CNRS, CFHT Corporation, 65-1238 Mamalahoa Hwy, Kamuela, HI 96743, USA

21 LESIA, Obs. de Paris, Place J. Janssen, 92195 Meudon Cedex, France

22 Rheinisches Institut für Umweltforschung an der Universität $\mathrm{zu}$ Köln, Aachener Strasse 209, 50931 Köln, Germany

23 Center for Astronomy and Astrophysics, TU Berlin, Hardenbergstr. 36, 10623 Berlin, Germany

${ }^{24}$ LUTH, Observatoire de Paris, CNRS, Université Paris Diderot, 5 place Jules Janssen, 92195 Meudon, France 